\title{
Cardiac resynchronization therapy (CRT) with right ventricular sense triggered left ventricular pacing benefits for the hemodynamics compared with standard CRT for chronic congestive heart failure: A cross-over study
}

\author{
Li-Jin $\mathrm{Pu}^{*}$, Yu Wang*, Ling Zhao, Zhi-Ling Luo, Bao-Tong Hua, Ming-Hua Han, \\ Shu-Min Li, Jun Yang, Lin Li, Yun-Zhu Peng, Tao Guo \\ Department of Cardiology, The First Affiliated Hospital of \\ Kunming Medical University, Kunming, China
}

\begin{abstract}
Background: The aim of this study was to investigate the effect of cardiac resynchronization therapy (CRT) with right ventricular (RV) sense triggered left ventricular (LV) pacing for chronic heart failure (CHF).

Methods: Thirty patients who were eligible for the Class I indication of CRT were enrolled and the informed consents were signed. Left ventricular ejection fraction (LVEF), diastolic mitral flow velocity time integral (VTI), mitral regurgitation flow VTI, and aortic valve flow VTI were measured with GE Vivid 7 (GE Medical, Milwaukee, WI, USA) before and after CRT. The echocardiographic measurements and the average annual costs of the device use were compared.

Results: The duration of QRS complex, the length of time used for optimization, and the average annual cost of the device use under RV sense triggered LV pacing were significantly less than that under standard biventricular (BiV) pacing $(p<0.01)$, while the average battery lifetime was longer. Subgroup analysis showed that LVEF, diastolic mitral flow VTI, and aortic valve flow VTI under RV sense triggered LV pacing were greater than that under standard BiV pacing with right or LV pre-activation. The average battery lifetime was significantly longer and the average annual cost of the device use was less. The mitral regurgitation flow VTI under RV sense triggered LV pacing was less than that under standard BiV pacing with RV pre-activation.
\end{abstract}

Conclusions: $R V$ sense triggered $L V$ provides benefits for CHF patients over standard CRT in terms of maintaining the physiological atrio-ventricular delay of atrio-ventricular node and improving the acute hemodynamic effects. (Cardiol J 2015; 22, 1: 80-86)

Key words: congestive heart failure, cardiac resynchronization therapy, right ventricular sense triggered left ventricular pacing

Address for correspondence: Tao Guo, MD, Department of Cardiology, The First Affiliated Hospital of Kunming Medical University, 295 Xi Chang Road, Kunming 650032, P.R. China, tel: +86 871 65324888, fax: +86 87165377618 , e-mail: guotao20@hotmail.com

Received: 06.09.2013 Accepted: 13.07.2014

*LiJin Pu and Yu Wang should be regarded as co-first authors. 


\section{Introduction}

Chronic heart failure (CHF) is a common disease with a high mortality in the patients with $\mathrm{CHF}$. Treatment of CHF has met some difficulty, especially at significant expense [1]. Conduction abnormalities, such as left bundle branch block (LBBB), frequently co-exist in patients with $\mathrm{CHF}$ [2]. LBBB, which manifests as dyssynchronization of left ventricle (LV) and right ventricle (RV), delay of LV contraction, and paradoxical movement of ventricular septum decreases the effective cardiac output [3].

By setting a relative shorter atrioventricular (AV) interval and an appropriate interventricular (VV) interval, cardiac resynchronization therapy (CRT) might realize AV and VV synchronization, mitigate mitral regurgitation, extend the time for ventricular filling, and restore the contribution of atrial contraction to LV filling. Furthermore, CRT can alleviate symptoms, reduce the rate of hospitalization, and significantly decrease mortality [4-6]. However, approximately $30 \%$ of patients remain non-responsive to CRT [7-9]. This phenomenon can be partly caused by the fixed short AV delay (AVD), which abolishes the physiological AVD of AV node (AVN), resulting in detrimental effects on cardiac structure and function. These may counteract the benefit of CRT. Therefore, setting appropriate AVD to maintain the physiological AVD of AVN and promote the conduction through the right His-Purkinje system, and pacing LV simultaneously might more better than standard biventricular (BiV) pacing. This study will evaluate the effect of RV sense triggered LV pacing, and compare it with standard BiV pacing.

\section{Methods}

\section{Patients}

Enrollment criteria. From September 2007 to September 2010, 30 CHF patients (7 females and 23 males) with LBBB, who were eligible for CRT implantation due to class I indication [8], were enrolled into this cross-over study at the First Affiliated Hospital of Kunming Medical University. The informed consents for the implantation of pacemaker or defibrillator with CRT function (INSYNC III 8042 CRT-P, INSYNC III PROTECT 7285 CRT-D, INSYNC MAXIMO 7304 CRT-D, and INSYNC SENTRY 7298 CRT-D, all produced by Metronic in USA) were obtained. The criteria of enrollment were: (i) ischemic or non-ischemic cardiomyopathy; (ii) New York Heart Association
Class III or ambulatory Class IV after optimal medical therapy for CHF; (iii) sinus rhythm; (iv) left ventricular ejection fraction (LVEF) $\leq 35 \%$, and (v) QRS $\geq 120 \mathrm{~ms}$. All the enrolled patients were given standard medical therapy for $\mathrm{CHF}$, including beta receptor antagonist, angiotensin converting enzyme inhibitors or angiotensin II receptor blocker, and spironolactone.

Exclusion criteria. Standards are as follows: (i) survival expectancy less than 1 year; (ii) cardiomyopathy due to reversible causes; (iii) valve diseases that have not addressed by surgery; (iv) hypertrophic obstructive cardiomyopathy; (v) right bundle branch block, and (vi) second or third degree AV block; (vii) $\mathrm{PQ}$ interval $>0.22 \mathrm{~s}$.

\section{Measurements}

Measurement of QRS duration. The electrocardiogram (ECG) data were recorded by MARQUET 12-lead ECG machine (GE, USA, speed: 25 $\mathrm{mm} / \mathrm{s}$; gain: $1 \mathrm{mV} / 10 \mathrm{~mm}$ ) before and after CRT (optimizing with current guideline and activating RV sense triggered LV pacing respectively). The QRS duration was automatically calculated by the machine.

Measurement of cardiac ultrasound indicators. The echocardiographic measurements were performed by a technician who was not aware of the group of patients. Firstly, LV end-diastolic diameter was assessed by Vivid 7 Color Doppler System (GE, USA, 2.5 MHz, Vivid 7). Secondly, diastolic mitral spectrum of blood flow was recorded with sample volume (pulse Doppler) on the level of mitral ostium under apex 4-chamber view. Thirdly, LV outflow tract spectrum of blood flow was recorded with sample volume (pulse Doppler) located $0.5 \mathrm{~cm}$ below the level of aortic valve under apex 5-chamber view. The following indicators were evaluated before and after CRT (optimizing with current guideline and activating RV sense triggered LV pacing, respectively) in each patient: (i) LVEF; (ii) diastolic mitral velocity time integral (VTI) and mitral regurgitation VTI, and (iii) aortic valve VTI. Each indicator was assessed 3 times and an average was calculated.

AV and VV interval optimization with traditional method. AV and VV intervals were optimized after CRT implantation. The optimal AVD was defined as the shortest AVD which could ensure the longest duration of ventricular filling, the largest stroke volume, and the minimization of mitral regurgitation. The pacing mode was programmed to $\mathrm{BiV}$ pacing $(\mathrm{RV}+\mathrm{LV})$ and the $\mathrm{AVD}$ was titrated. If the function of sinus node of the 
patient was normal, then sensed AV interval was used. If the percentage of atrial pacing was greater than $50 \%$, then the paced AV interval was used. The aortic spectrum of blood flow was recorded under apex 5-chamber view and the aortic VTI was calculated. The optimal AV interval was confirmed when the largest aortic VTI, the minimization of mitral regurgitation, the longest duration of ventricular filling, and the highest peak of separated intact $\mathrm{E}$ and $\mathrm{A}$ wave were accomplished $[10,11]$. $\mathrm{VV}$ interval was optimized after the AV interval optimization was completed. The optimal VV interval was confirmed when the largest aortic VTI was accomplished [12-14]. The cardiac function corresponding to each $\mathrm{AV}$ interval was calculated 5 min after the AV interval was titrated. The echocardiographic measurements of each patient were recorded after the AV interval and the VV intervals were optimized.

The programming of RV sense triggered LV pacing. The electrogram marker channel and the ECG were showed on the programmer (9790, Medtronic, USA). The AVD was extended step by step until ventricular sense showed upon the marker channel. The pacing mode was programmed to LV-only, meanwhile, the ventricular sense reaction (VSR) was set to $\mathrm{ON}$, i.e. activating RV sense triggered LV pacing. LVEF, diastolic mitral VTI, mitral regurgitation VTI, and aortic VTI were assessed 5 min later. The optimal AV and VV intervals were restored after the previously mentioned measurements were completed. The time (minute as unit) for optimizing the 2 different CRT pacing modes was recorded respectively.

Estimate of the battery lifetime. The battery lifetime was estimated under standard $\mathrm{BiV}$ pacing and RV sense triggered LV pacing, respectively. The minimal, maximal, and average battery lifetimes were automatically calculated by the system. The average annual cost of the device use was calculated according to the cost of CRT device and the estimated battery lifetime.

\section{Data analysis}

All data were analyzed with SPSS 13.0 software. Continuous variables were expressed as mean and standard deviation. Data in the three groups were compared with variance analysis (Dunnet's test was used if variance was not homogeneous). The means in two groups were compared with $t$ test if the data were conformed as normal distribution; if not, Mann-Whitney U test was used. $\mathrm{P}<0.05$ was considered statistically significant.

\section{Results}

The duration of QRS complex $(131.45 \pm 7.48 \mathrm{~ms})$, the length of time used for optimization (10.79 \pm $\pm 4.02 \mathrm{~min}$ ), and the average annual cost of the device use under RV sense triggered $L V$ pacing [mean 13800 (13100-14500) Ren Min Bi (RMB)] were significantly lower than those under standard $\mathrm{BiV}$ pacing $(139.76 \pm 11.73 \mathrm{~ms}, 55.6 \pm 8.77 \mathrm{~min}$, and mean 21200 (20400-22000) RMB, respectively $(\mathrm{p}<0.01)$.

The comparison of measurements prior to CRT, standard $\mathrm{BiV}$ and pacing $\mathrm{RV}$ sense triggered $\mathrm{LV}$ pacing treatment was displayed in Table 1 . There were no significant differences of LVEF, diastolic mitral flow VTI, mitral regurgitation flow VTI, and aortic valve flow VTI between RV sense triggered $\mathrm{LV}$ pacing and standard BiV pacing ( $\mathrm{p}>0.05$ ). Figure 1 shows the ECG of a patient with dilated cardiomyopathy before treatment and after tre-

Table 1. The comparison of measurements between baseline, standard biventricular (BiV) pacing and right ventricular (RV) sense triggered left ventricular $(L V)$ pacing $(n=30)$.

\begin{tabular}{lccc}
\hline Measurements & Prior to CRT & Standard BiV pacing & RV sense triggered LV pacing \\
\hline Atrioventricular delay [ms] & $145.28 \pm 5.69^{\&}$ & $123.17 \pm 5.55^{\#}$ & $186.90 \pm 7.36^{\# *}$ \\
QRS duration [ms] & $152.93 \pm 17.07$ & $139.76 \pm 11.73^{\#}$ & $131.45 \pm 7.48^{\# *}$ \\
LVEF [\%] & $26.86 \pm 6.09$ & $35.03 \pm 5.08^{\#}$ & $34.76 \pm 5.30^{\#}$ \\
Mitral VTI & $16.49 \pm 1.14$ & $18.69 \pm 1.31^{\#}$ & $18.42 \pm 1.11^{\#}$ \\
Mitral regurgitation VTI & $155.72 \pm 23.07$ & $135.62 \pm 19.88^{\#}$ & $137.07 \pm 17.72^{\#}$ \\
Aortic VTI & $17.20 \pm 1.13$ & $19.36 \pm 1.06^{\#}$ & $19.17 \pm 1.14^{\#}$ \\
Time for optimization [min] & - & $55.6 \pm 8.77$ & $10.79 \pm 4.02^{*}$ \\
Average battery lifetime [yeas] & - & $4.25 \pm 0.16$ & $6.55 \pm 0.35^{*}$ \\
Average annual cost of the & - & $21.2 \pm 0.80$ & $13.80 \pm 0.70^{*}$ \\
device use (thousand RMB) & & & \\
\hline
\end{tabular}

${ }^{*} p<0.01$ compared with standard BiV pacing; ${ }^{*} p<0.01$ compared with prior to CRT; ${ }^{\mathrm{P} Q}$ interval; CRT — cardiac resynchronization therapy; LVEF — left ventricular ejection fraction; VTI - velocity time integral; RMB — Ren Min Bi 


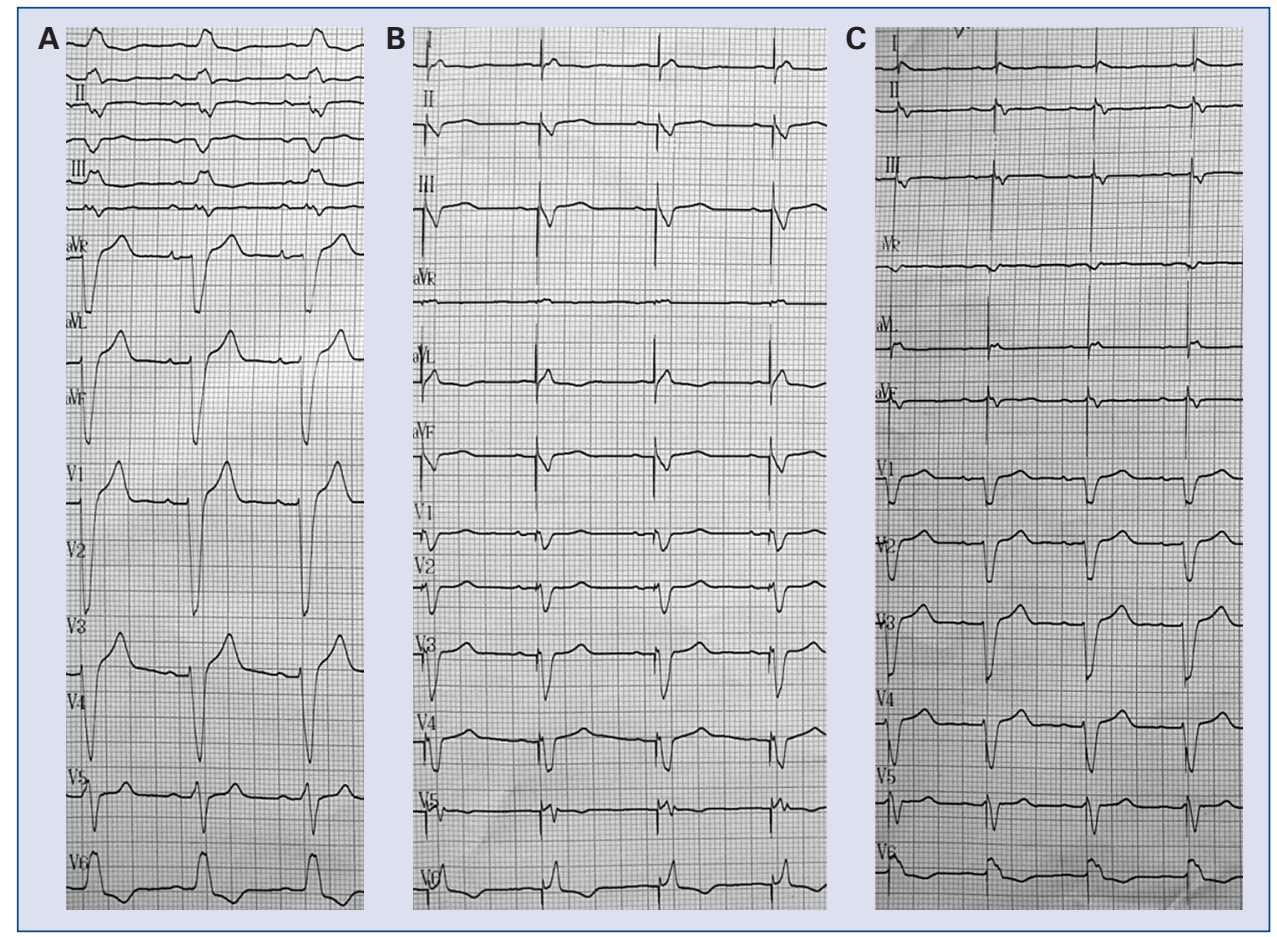

Figure 1. Registration of 12-lead electrocardiogram of a patient with dilated cardiomyopathy: A. The patient was in sinus rhythm with complete left bundle branch block, and the QRS duration was 0.16 s prior to cardiac resynchronization therapy implantation; B. Standard biventricular (BiV) pacing with VV delay of $10 \mathrm{~ms}$ (the optimized AV delay was $130 \mathrm{~ms}$, QRS duration was $0.14 \mathrm{~s}$ ); C. Right ventricular sense triggered left ventricular pacing (the intrinsic PR interval was $200 \mathrm{~ms}$, the sensed AV was set at $220 \mathrm{~ms}$ and QRS duration was $0.12 \mathrm{~s}$ which was significantly narrower than that of standard BiV pacing). The electrocardiograms on leads I, II, III, AVF, and $V_{4}-V_{6}$ showed that the stimulation was released at the beginning of $R$ wave.

atment with standard BiV and pacing RV sense triggered LV pacing.

Subgroup analysis is displayed in Table 2 and the results showed that LVEF, mitral VTI, aortic VTI, and average battery lifetime under RV sense triggered $L V$ pacing were significantly greater than those under the standard BiV pacing with RV pre-activation. QRS duration, mitral regurgitation flow VTI, time for optimization, and the average annual cost of the device use under RV sense triggered $\mathrm{LV}$ were significantly lower than those under the standard BiV pacing with RV pre-activation $(\mathrm{p}<0.01$ or $\mathrm{p}<0.05)$.

QRS duration, LVEF, mitral VTI, mitral regurgitation flow VTI and aortic VTI between standard BiV pacing with LV pre-activation and RV sense triggered LV pacing are shown in Table 3. There were no significant differences for these parameters. Time for optimization and the average annual cost of the device use in RV sense triggered $\mathrm{LV}$ pacing were lower than those of standard $\mathrm{BiV}$ pacing with LV pacing pre-activation $(\mathrm{p}<0.01)$.
Average battery lifetime in RV sense triggered LV pacing was longer than that of standard $\mathrm{BiV}$ pacing with $\mathrm{LV}$ pacing pre-activation $(\mathrm{p}<0.01)$.

In addition, the subgroup analysis showed that the acute hemodynamic effects of the RV sense triggered LV pacing were better than those of the standard BiV pacing after excluding the patients who need pacing the LV first.

\section{Discussion}

Chronic heart failure is one of the most common diseases with high mortality. Many studies have been conducted to investigate its clinical traits, mechanism, and therapy. The present study concentrated on the CRT with RV sense triggered LV pacing, and the difference of acute hemodynamic effects between standard BiV pacing and RV sense trigged LV pacing. The subgroup analysis indicated that CRT with the RV sense trigged LV was superior to the standard BiV pacing with $\mathrm{RV}$ pre-activation. 
Table 2. The comparison of measurements between standard biventricular (BiV) pacing with right ventricular (RV) pacing pre-activation and RV sense triggered left ventricular (LV) pacing $(n=11)$.

\begin{tabular}{lcc}
\hline Measurements & Standard BiV pacing with RV pre-activation & RV sense triggered LV pacing \\
\hline Atrioventricular delay [ms] & $122.14 \pm 5.79$ & $187.14 \pm 7.76^{*}$ \\
QRS duration [ms] & $140.79 \pm 8.89$ & $128.07 \pm 2.73^{*}$ \\
LVEF [\%] & $33.5 \pm 5.37$ & $37.43 \pm 4.03^{\#}$ \\
Mitral VTI & $18.11 \pm 1.27$ & $19.27 \pm 1.32^{\#}$ \\
Mitral regurgitation VTI & $134.36 \pm 20.21$ & $120.36 \pm 12.34^{\#}$ \\
Aortic VTI & $18.84 \pm 0.88$ & $19.71 \pm 0.83^{\#}$ \\
Time for optimization [min] & $56.79 \pm 9.14$ & $11.86 \pm 4.47^{*}$ \\
Average battery lifetime [yeas] & $4.24 \pm 0.16$ & $6.58 \pm 0.34^{*}$ \\
Average annual cost of the & $21.30 \pm 0.80$ & $13,70 \pm 0.70^{*}$ \\
device use (thousand RMB) & & \\
\hline
\end{tabular}

${ }^{*} p<0.01$ compared with RV pre-activation; ${ }^{*} p<0.05$ compared with RV pre-activation; LVEF — left ventricular ejection fraction; VTI — velocity time integral; RMB - Ren Min Bi

Table 3. The comparison of measurements between standard biventricular (BiV) pacing with left ventricular (LV) pacing pre-activation and right ventricular (RV) sense triggered LV pacing $(n=19)$.

\begin{tabular}{lcc}
\hline Measurements & Standard BiV pacing with LV pre-activation & RV sense triggered LV pacing \\
\hline Atrioventricular delay [ms] & $124.20 \pm 5.31$ & $186.40 \pm 6.96^{*}$ \\
QRS duration [ms] & $138.73 \pm 14.57$ & $134.07 \pm 7.34$ \\
LVEF [\%] & $36.56 \pm 4.79$ & $32.09 \pm 6.57^{\#}$ \\
Mitral VTI & $19.30 \pm 1.35$ & $19.27 \pm 0.90$ \\
Mitral regurgitation VTI & $136.88 \pm 19.55$ & $153.78 \pm 23.10^{\#}$ \\
Aortic VTI & $19.88 \pm 1.24$ & $18.63 \pm 1.45^{*}$ \\
Time for optimization [min] & $54.41 \pm 8.40$ & $9.70 \pm 3.57^{*}$ \\
Average battery lifetime [yeas] & $4.26 \pm 0.16$ & $6.52 \pm 0.36^{*}$ \\
Average annual cost of the & $21.10 \pm 0.80$ & $13.90 \pm 0.70^{*}$ \\
device use (thousand RMB) & &
\end{tabular}

${ }^{*} p<0.01$ compared with LV pre-activation; ${ }^{*} p<0.05$ compared with LV pre-activation; LVEF — left ventricular ejection fraction; VTI — velocity time integral; RMB — Ren Min Bi

Supraventricular pulses rapidly conduct through His-Purkinje system via AVN to activate ventricles. However, the pulses slowly conduct through cardiomyocytes and retrograde to the base of heart via His-Purkinje system along ventricular septum when pacing from RV apex. The changed activation sequence might cause the abnormality of the structure of cardiomyocytes, disarrangement of myocardial fibers, the damage of the structure of mitochondria, and myocardial calcification. These will influence the cell structure and ventricular geometry, and even the synchrony of ventricular contraction and relaxation. These variations might further harm the blood bump function of the ventricles. Therefore, minimized percentage of ventricular pacing (MVP) can decrease the risk of persistent atrial fibrillation [15]. In order to capture both ventricles during traditional CRT, AVD is often set to a relatively short value (100-120 ms) $[16,17]$. Previous studies indicate that too short AVD will decrease the cardiac output of the patients with $\mathrm{CHF}$, furthermore, the ventricular filling is more sufficient when AVD is programmed to 200-250 ms. Under physiological condition, the optimal AVD might be affected by influence factors, such as exercise, sympathetic tone change and heart rate $[11,18,19]$. Generally, the PR interval will be prolonged $4 \mathrm{~ms}$ when the heart rate decreases every 10 beats in order to coordinate the function of atrium with ventricular filling. When there is no AV block [12], the intrinsic PR interval itself may be the optimal AVD. The cumbersome AVD optimization is unsuitable to perform at any time under the guidance of echocardiography [20]. Therefore, setting appropriate AVD, encouraging AVN preference to promote the conduction through the right His- 
-Purkinje system via dynamic intrinsic PR interval, and pacing LV simultaneously might meet the principles of physiological pacing, and further improve the effectiveness of CRT in patients with LBBB.

Three-chamber pacemaker with VSR invented by Medtronic provides another solution for the patients who have a conduction of intrinsic rhythm (such as atrial fibrillation). CRT with RV sense triggering is the solution for LV pacing to attain CRT. In the present study, the results indicated that CRT could be realized by VSR pacing that sensed $\mathrm{R}$ wave by the electrodes located in the $\mathrm{RV}$ and trigger the LV pacing simultaneously when the intrinsic rhythms conduct to the RV. Thus, AVN preference was ensured and the principles of physiological pacing such as dynamic AVD and MVP were followed. In addition, since there was no need to pace the RV, the estimated battery lifetime was relatively extended and the average annual cost of the device use was indirectly decreased. Generally, the warranty period of the pacemaker under standard BiV pacing is 4 years. In this study, the battery lifetime could be extended to another 2 years, and the average annual lifetime related costs of CRT device only could be decreased (from $21200 \mathrm{RMB}$ to $13800 \mathrm{RMB}$ ) under the RV sense triggered LV pacing ( $\mathrm{p}<0.01)$. However, many factors (such as artificial parameter and category of instrument) may affect the metrics of battery lifetime. Thus, a further investigation is needed to confirm this conclusion.

The activation sequence of the RV might be restored since the supraventricular pulses rapidly conduct to the RV under the RV sense triggered $\mathrm{LV}$ pacing. The time for RV activation was longer under the standard BiV pacing than that for the physiological activation because the activation was slowly conducted retrograde through cardiomyocytes via His-Purkinje system, which manifests as longer $\mathrm{QRS}$ duration $(\mathrm{p}<0.01)$. The former researches have proved that the abnormality of intraventricular conduction manifests as the extension of QRS duration and that the QRS duration is associated with mechanical dyssynchrony, i.e. the longer the QRS duration, the higher the percentage of dyssynchrony, which means lower cardiac function and higher mortality [21]. The present study showed that RV sense triggered LV pacing may shorten the QRS duration in patients with $\mathrm{CHF}$ compared with standard BiV pacing. $\mathrm{AV}$ and VV interval optimization was time-consuming in standard BiV pacing, which generally took about $1 \mathrm{~h}$ every time and may increase the cost. Moreover, it was difficult to perform the optimization in pra- ctice due to the effect of the position of patients, the angle of ultrasound probe, and the ability of technicians, which all lead to poor repeatability of the echocardiographic measurements. Thus, it was difficult to achieve individualization and dynamic optimization. In addition, the indicators and standards are still unclear [22, 23]. However, when the RV sense triggered LV pacing was programmed, the intrinsic PR interval itself might be the optimal AVD.

\section{Limitations of the study}

There were still some limitations to the present study, such as the pacing of the RV was earlier than the LV to some extent under the RV sense triggered LV pacing. Moreover, the degree of advancement and the release of the stimulus for the LV depended on the slew rate of the RV electrogram. Theoretically, patients who need pacing the LV first might not benefit from the RV sense triggered LV pacing. However, the previous studies found that about one-third of patients with $\mathrm{CHF}$ need pacing the RV first when the optimization of VV interval is performed [24]. The initial values of $\mathrm{AV}$ and $\mathrm{VV}$ are suggested to be programmed. Guidelines for AV and VV programming for $\mathrm{CHF}$ patients have been put forward [25, 26]. There was no significant difference of the acute hemodynamic effects between the RV sense triggered LV pacing and standard $\mathrm{BiV}$ pacing in this study. The reason might be related to a small sample size and inclusion of the patients who need pacing the LV first.

The subgroup analysis showed that the acute hemodynamic effects of the RV sense triggered LV pacing were better than those of the standard BiV pacing after excluding the patients who need pacing the LV first. Thus, the optimal indication for the RV sense triggered LV pacing might be suited for patients who have no response to the standard BiV pacing and the patients who need pacing the RV first. The results of this study showed that the RV sense triggered LV pacing could improve acute hemodynamic effects of the patients with $\mathrm{CHF}$ compared with the standard BiV pacing. This algorithm might cause dispute between maintenance and abolishment of the physiological AVD of AVN in current CRT, as well as even challenge to subvert the recommendation of $100 \% \mathrm{BiV}$ pacing in current guidelines. However, a randomized, double blind, controlled clinical research approved by ethics committee is needed to compare the improvement of cardiac function and clinical outcome with respect to two different pacing modes in the next research. 


\section{Conclusions}

Right ventricular sense triggered LV provides benefits to CHF patients over standard CRT in terms of maintaining the physiological AVD of AVN and improving the acute hemodynamic effects.

Funding sources: This study was supported by a grant from Yunnan Science and Technology Committee (2009CD158).

\section{Conflict of interest: None declared}

\section{References}

1. O'connell J. The economic burden of heart failure. Clin Cardiol, 2000; 23: III6-III10.

2. Jurcut R, Pop I, Calin C, Coman IM, Ciudin R, Ginghina C. Utility of QRS width and echocardiography parameters in an integrative algorithm for selecting heart failure patients with cardiac dyssynchrony. Eur J Internal Med, 2009; 20: 213-220.

3. Grines CL, Bashore T, Boudoulas H, Olson S, Shafer P, Wooley C. Functional abnormalities in isolated left bundle branch block. The effect of interventricular asynchrony. Circulation, 1989; 79: 845-853.

4. Auricchio A, Stellbrink C, Block M et al. Effect of pacing chamber and atrioventricular delay on acute systolic function of paced patients with congestive heart failure. Circulation, 1999; 99: 2993-3001.

5. Kass DA, Chen C-H, Curry C et al. Improved left ventricular mechanics from acute VDD pacing in patients with dilated cardiomyopathy and ventricular conduction delay. Circulation, 1999; 99: 1567-1573.

6. Ukkonen H, Beanlands RS, Burwash IG et al. Effect of cardiac resynchronization on myocardial efficiency and regional oxidative metabolism. Circulation, 2003; 107: 28-31.

7. Epstein AE, DiMarco JP, Ellenbogen KA et al. ACC/AHA/HRS 2008 Guidelines for Device-Based Therapy of Cardiac Rhythm Abnormalities: A Report of the American College of Cardiology/ American Heart Association Task Force on Practice Guidelines (Writing Committee to Revise the ACC/AHA/NASPE 2002 Guideline Update for Implantation of Cardiac Pacemakers and Antiarrhythmia Devices) Developed in Collaboration With the American Association for Thoracic Surgery and Society of Thoracic Surgeons. J Am Coll Cardiol, 2008; 51: e1-e62.

8. Hunt. ACC/AHA 2005 guideline update for the diagnosis and management of chronic heart failure in the adult: A report of the American College of Cardiology/American Heart Association task force on practice guidelines (Writing Committee to update the 2001 guidelines for the evaluation and management of heart failure)(vol 46, pg e1, 2005). J Am Coll Cardiol, 2006; 47: 1503-1505.

9. Yu CM, Wing Hong Fung J, Zhang Q, Sanderson JE. Understanding nonresponders of cardiac resynchronization therapy: Current and future perspectives. J Cardiovasc Electrophysiol, 2005; 16: 1117-1124.

10. Meluzn J, Novæk M, Mllerova J et al. A fast and simple echocardiographic method of determination of the optimal atrioventricular delay in patients after biventricular stimulation. Pacing Clin Electrophysiol, 2004; 27: 58-64.
11. Scharf C, Li P, Muntwyler Jr, Chugh A et al. Rate-dependent AV delay optimization in cardiac resynchronization therapy. Pacing Clin Electrophysiol, 2005; 28: 279-284.

12. Bordachar P, Lafitte S, Reuter S et al. Echocardiographic parameters of ventricular dyssynchrony validation in patients with heart failure using sequential biventricular pacing. J Am Coll Cardiol, 2004; 44: 2157-2165.

13. Mortensen PT, Sogaard P, Mansour H et al. Sequential biventricular pacing. Pacing Clin Electrophysiol, 2004; 27: 339-345.

14. Vanderheyden M, De Backer T, Rivero-Ayerza $\mathrm{M}$ et al. Tailored echocardiographic interventricular delay programming further optimizes left ventricular performance after cardiac resynchronization therapy. Heart Rhythm, 2005; 2: 1066-1072.

15. Cleland JG, Coletta AP, Abdellah AT, Witte KK, Hobson N, Clark AL. Clinical trials update from Heart Rhythm 2007 and Heart Failure 2007: CARISMA, PREPARE, DAVID II, SAVE-PACE, PROTECT and AREA-IN-CHF. Eur J Heart Failure, 2007; 9: 850-853.

16. Riedlbauchova L, Kautzner J, Fridl P. Influence of different atrioventricular and interventricular delays on cardiac output during cardiac resynchronization therapy. Pacing Clin Electrophysiol, 2005; 28: S19-S23.

17. Ritter P, Padeletti L, Gillio-Meina L, Gaggini G. Determination of the optimal atrioventricular delay in DDD pacing Comparison between echo and peak endocardial acceleration measurements. Europace, 1999; 1: 126-130.

18. O'Donnel D, Nadurata V, Hamer A, Kertes P, Mohammed U. Long-term variations in optimal programming of cardiac resynchronization therapy devices. Pacing Clin Electrophysiol, 2005; 28: S24-S26.

19. Mokrani B, Lafitte S, Deplagne A et al. Echocardiographic study of the optimal atrioventricular delay at rest and during exercise in recipients of cardiac resynchronization therapy systems. Heart Rhythm, 2009; 6: 972-977.

20. Gras D, Gupta MS, Boulogne E, Guzzo L, Abraham WT. Optimization of AV and VV Delays in the Real-World CRT Patient Population: An International Survey on Current Clinical Practice. Pacing Clin Electrophysiol, 2009; 32: S236-S239.

21. Iuliano S, Fisher SG, Karasik PE, Fletcher RD, Singh SN. QRS duration and mortality in patients with congestive heart failure. Am Heart J, 2002; 143: 1085-1091.

22. Harris S, Tepper D, Ip R. Comparison of the effects of cardiac resynchronization therapy in patients with class II vs class III and IV heart failure (From the InSync/InSync ICD Italian Registry). Congestive Heart Failure, 2008; 14: 111.

23. Miki Y, Ishikawa T, Matsushita K et al. Optimal programming of the atrioventricular delay using the phonocardiogram. Pacing Clin Electrophysiol, 2009; 32: S81-S85.

24. Dougherty AH, Naccarelli GV, Gray EL, Hicks CH, Goldstein RA. Congestive heart failure with normal systolic function. Am J Cardiol, 1984; 54: 778-782.

25. Parkash R, Philippon F, Shanks M et al. Canadian cardiovascular society guidelines on the use of cardiac resynchronization therapy: Implementation. Canadian J Cardiol, 2013; 29: 1346-1360.

26. Members ATF, Brignole M, Auricchio A et al. 2013 ESC Guidelines on cardiac pacing and cardiac resynchronization therapy: The Task Force on cardiac pacing and resynchronization therapy of the European Society of Cardiology (ESC). Developed in collaboration with the European Heart Rhythm Association (EHRA). Eur Heart J, 2013; 34: 2281-2329. 